\title{
Neural network technique when distribution of vehicle component parts on the technological repair routes, taking into account their technical condition
}

\author{
Valentin Krasovsky ${ }^{1}$, Nina Krasovskaya ${ }^{1}$, Victor Poptsov ${ }^{1}$, and Irina Nordman ${ }^{1, *}$ \\ ${ }^{1}$ Industrial University of Tyumen, 625000, 38, Volodarskogo Str., Tyumen, Russia
}

\begin{abstract}
Increase of repair efficiency is achieved due to the formation of centralized specialized production facilities which implement the vehicle component parts repair technique with the use of industrial technological processes to restore the technical state of the units and their components. In this case, the establishment of the expediency of sending the unit to repair, as well as the defining of volumes and nomenclature for necessary repair actions, should be performed at the stage of pre-repair diagnosis for each individual unit taking into account its actual technical condition. However, the effectiveness of pre-repair diagnosis using both deterministic and probabilistic methods of processing and analyzing the information obtained is significantly reduced by the presence of errors in the recognition of defects and the distribution of aggregates in accordance with the repair work variety preformed at the repair enterprise. Using promising cognitive technology based on neural networks it is possible to completely avoid the losses associated with the repetition of repair work. Therefore, the formation of scientific and methodological bases for the development, training and practical application of artificial neural networks in the subsystems of the pre-repair diagnosis of the repair fund of automobile vehicle omponent parts is an important and urgent task. The paper presents the results of analytical studies and a number of original techniques for the formation of scientific and methodological foundations for the development, training and practical application of artificial neural networks in the process of diagnosis the car vehicle component parts and special oil and gas equipment entering the centralized repair according to their technical condition
\end{abstract}

\section{Introduction}

The effectiveness of pre-repair diagnosis using both deterministic and probabilistic methods of processing and analyzing the information obtained is significantly reduced by the presence of errors in the recognition of defects and the distribution of vehicle component parts in accordance with the repair work variety [1,2]. Using the advanced cognitive

\footnotetext{
* Corresponding author: nordman.i@inbox.ru
} 
technology based on artificial neural networks it is possible to completely avoid the losses associated with the repetition of the repair work. Therefore, the formation of scientific and methodological foundations for the development, training and practical application of artificial neural networks in the process of diagnosis the vehicle component parts and special oil and gas equipment entering the centralized repair according to the technical condition is an important and urgent task.

\subsection{Use of artificial neural networks to solve the problem of identifying the technical state of aggregates and the distribution of the repair work variety}

A set of methods for the intellectual analysis of research object data was called Data Mining. The knowledge produced by these methods is usually presented in the form of models. Artificial neural networks are one such class of models. These are mathematical models that represent an ordered set of artificial neurons related to each other in a certain way.

The expediency of using an artificial neural network as a mathematical device for solving problems of identifying the technical condition of vehicle component parts of a repair fund and their distribution by repair work variety under the centralized repair according to the technical condition is due to a number of reasons [3].

1. Techniques built on the basis of this mathematical apparatus can effectively solve the research tasks.

2. The technique built on the basis of an artificial neural network is an effective device for determining the technical condition of car vehicle component parts and special oil and gas equipment entering the repair fund, while permitting the elimination of recognition errors of the 1 st and 2 nd type.

3. The use of an artificial neural network makes it possible to exclude the need to accumulate statistical information to optimize the distribution of vehicle component parts for repair work.

4. It is very easy to verify the adequacy of models constructed on the basis of an artificial neural network using test samples formed during the experiment on the research object.

A multilayer perceptron is one of the varieties of a neural network. It is the union of neurons in interconnected layers. In the years 1987-1991, professor of the University of California (USA) R. Hecht-Nielsen revised Arnold-Kolmogorov's theorems for artificial neural networks. He proved that for any set of distinct pairs (between which there were no contradictions) of arbitrary dimension $\left(\mathrm{X}_{\mathrm{q}}, \mathrm{D}_{\mathrm{q}}\right), \mathrm{q}=\{1, \ldots, \mathrm{Q}\}$, there was a two-layer perceptron with sigmoid activation functions and a finite number of neurons, which forms the corresponding output vector $\mathrm{D}_{\mathrm{q}}$ for each input vector $\mathrm{X}_{\mathrm{q}}[4]$.

When using artificial neural networks, the question arises first of all of the choice of the network architecture for a specific task (the number of "layers" and the number of "artificial neurons" in each of them).

An artificial neuron is an artificial neural networks node modeled on the simplified principle of functioning of a biological neuron. The article of W. McCullock and W. Pitts, published in 1943, is considered as the first paper, laid the theoretical foundations for the creation of intelligent devices. The first translation into Russian was published in 1956 [5].

From the mathematical point of view, the artificial neuron is a function of a single argument - a linear combination of all signals at the input (this function was called the "activation function"), which the output of the neuron is generated through.

In general, the mathematical model of an artificial neuron is a weighted adder and has the form: 


$$
S=\sum_{i=1}^{n} x_{i} \cdot w_{i}+x_{0} \cdot w_{0}=\sum_{i=0}^{n} x_{i} \cdot w_{i}
$$

where $\mathrm{S}$ - weighted sum of input signals of a neuron; $\boldsymbol{x}_{i}$ - value at the $\mathrm{i}^{\text {th }}$ input of a neuron; $w_{i}$ - weight of $\mathrm{i}^{\text {th }}$ synapse; $\boldsymbol{n}$ - number of inputs; $\boldsymbol{x}_{0}$ and $\boldsymbol{w}_{0}$ - respectively, values of the additional input signal $\left(\mathrm{X}_{0}=1\right)$ and its weight respectively.

The output value of a neuron is a function of its state:

$$
Y=f(S)
$$

where $\quad f(S)$ is an activation function.

All layers of the neural network can be conditionally divided into three groups:

- the first layer of neurons in a multilayer neural network is called an input layer. No computational operations usually are performed in it as it consists of neurons which serve to obtain data (signals) and further transfer to the inputs of the hidden layer of artificial neural networks;

- hidden (intermediate) layers are the main ones as they most often form a large part of the artificial neural networks structure. Calculations in them are made by the formulas (1) and (2);

- the output layer is the result of the network operation.

The choice of a sigmoid as an activation function is determined by the fact that it is differentiable along the whole axis of abscissas and has a very simple derivative. When using the back propagation error algorithm, this helps to speed up the learning process of the network $[6,7]$.

The steepness $\alpha$ is the main parameter which the form of the sigmoid function depends on.

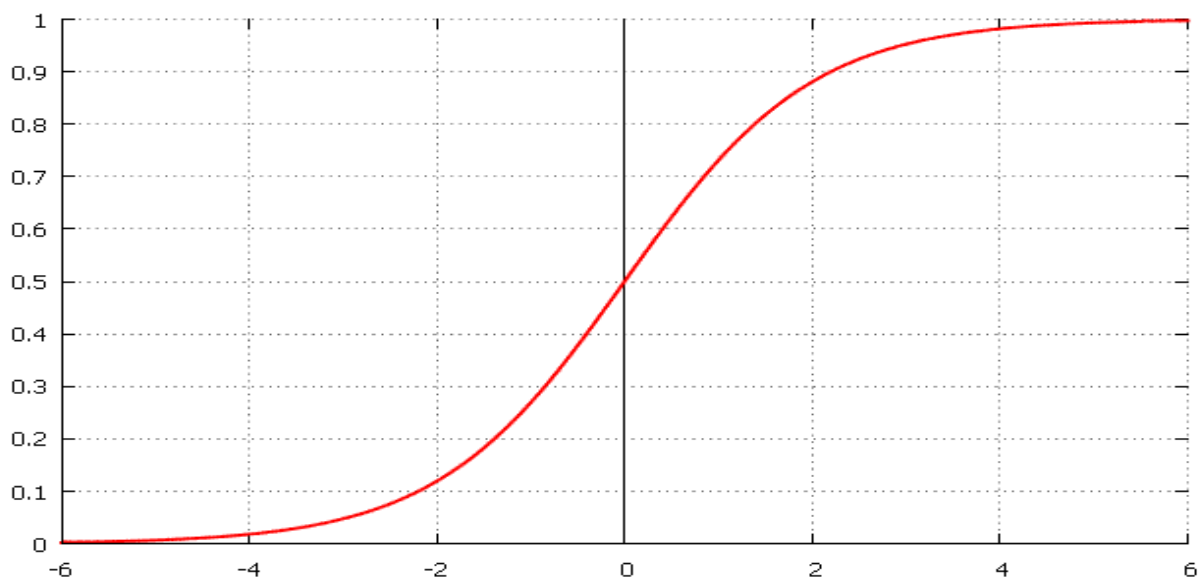

Fig. 1. Sigmoid function when $\alpha=1.0$ 


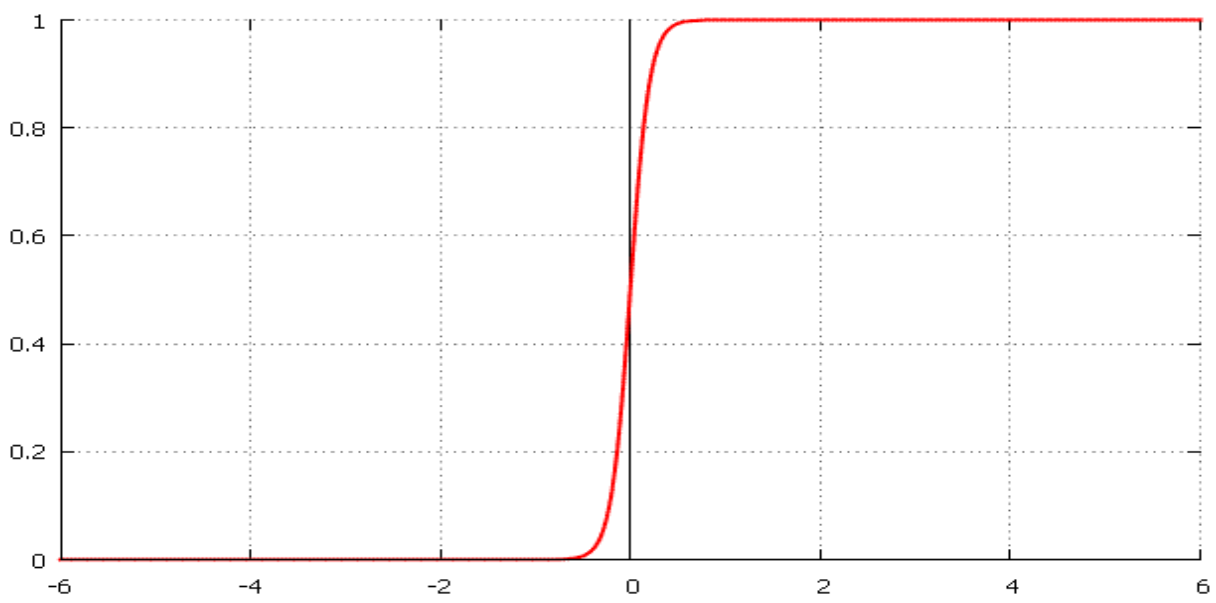

Fig. 2. Sigmoid function when $\alpha=0.1$

As the parameter $\alpha$ decreases the sigmoid takes the form of a linear function (Figure 2), but as the steepness increases, it approaches the Heaviside function (the function of a single jump of the "step" type) (Fig. 3).

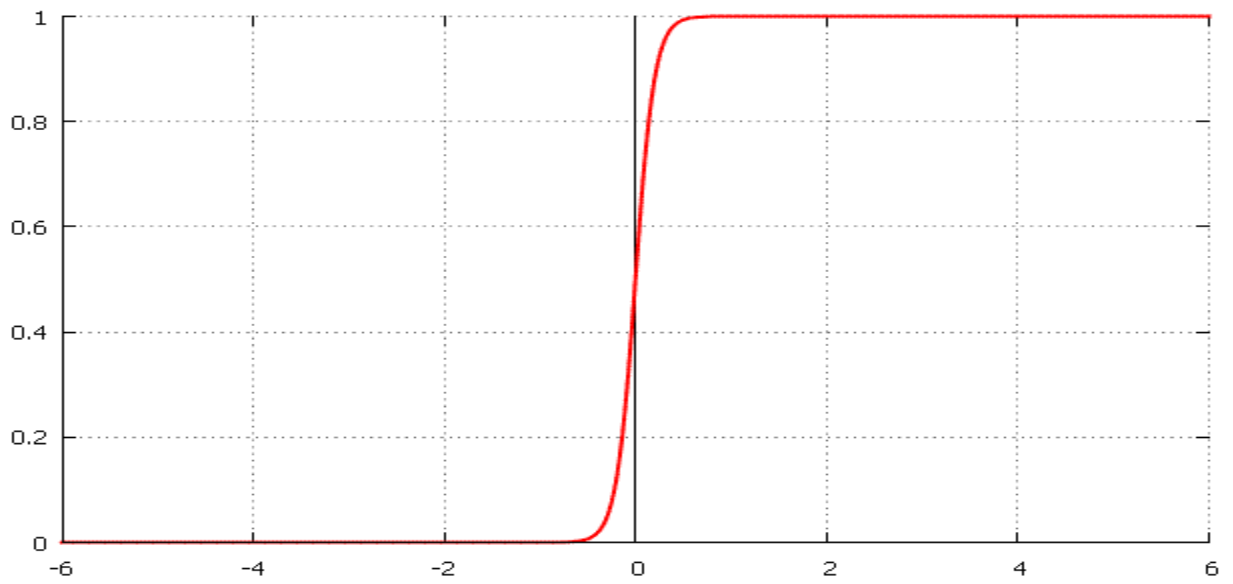

Fig. 3. Sigmoid function when $\alpha=10$

The output value of a neuron, with a sigmoidal activation function acquires the following form:

$$
Y=f(S)=\frac{1}{1+e^{-\alpha S}}
$$

In Fig. 4 graphically represents a model of an artificial neuron, where the number of input signals is denoted as $\mathrm{X}$. Here, multiple signals $x_{1}, x_{2}, x_{3}, \ldots, x_{j}$ at the corresponding inputs (collectively denoted by the vector $\mathrm{X}$ ) have their weights (which reflect the strength of the synoptic connection and their set is denoted by the vector $\mathrm{W}$ ). The product of the signals and the corresponding weights is fed to the summing unit, which algebraically adds the inputs. 


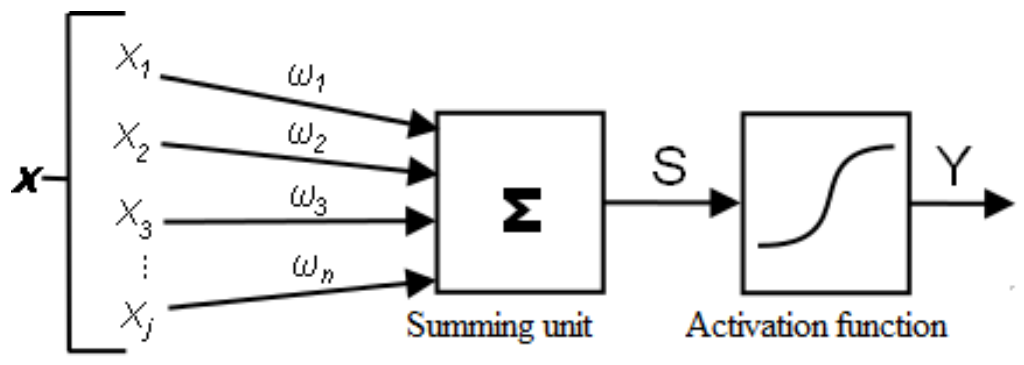

Fig. 4. Model of an artificial neuron

As a result of the accumulation, the resulting value is the argument of the activation function, which creates the output value Y.

Proceeding from theoretical studies by Hecht-Nielsen on the optimal number of hidden layers, and also from the analysis of the practical applicability of artificial neural networks for various classification problems, it can be concluded that it is most often impractical to use of more than two hidden layers in the design of the network.

By the formulas (4) and (5), which is a consequence of the Arnold-Kolmogorov-HechtNielsen theorems, it is possible to calculate the required number of neurons for the hidden layer.

At the beginning, the necessary number of weights of the synaptic connection is estimated:

$$
\frac{N_{y} \cdot Q}{1+\log _{2} Q} \leq N_{w} \leq N_{y} \cdot\left(\frac{Q}{N_{x}}+1\right) \cdot\left(N_{x}+N_{y}+1\right)+N_{y}
$$

where $N_{y}$ - output signal dimensionality; Q - number of elements in the training sample; $N_{w}$ - necessary number of synaptic weights; $N_{x}$-input signal dimension.

After this, the number of neurons in the hidden layer will be determined by the formula:

$$
\boldsymbol{L}=\frac{\boldsymbol{N}_{w}}{\boldsymbol{N}_{x}+\boldsymbol{N}_{y}}
$$

However, as the practice of constructing artificial neural networks shows, it cannot be asserted with this approach that the number of neurons in the hidden layer was optimally matched to the problem posed. in addition, as a result, a large range of the interval in which the value lies $L$ is obtained.

Therefore, the corollary of the Arnol'd-Kolmogorov-Hecht-Nielsen theorems will be used only to determine the upper limit of the value of the neurons $(\mathrm{R})$ in the hidden layer. Discarding the lower bound of the interval in (4) and equating $\mathrm{N}_{\mathrm{w}}$ to the remaining upper bound, we substitute formula (5) in (4) and obtain:

$$
R=\frac{N_{y}\left(\frac{Q}{N_{x}}+1\right)\left(N_{x}+N_{y}+1\right)+N_{y}}{N_{x}+N_{y}}
$$

Further, $\mathrm{R}$ will be used as an upper limit, to which the number of neurons will be increased until it reaches the optimal value.

As the experience of building models based on artificial neural networks shows, building up neurons in a hidden layer above the obtained $\mathrm{R}$ limit is in most cases impractical. 


\section{Methods}

Techniques of neural network classification of the repair fund depending on the identified technical condition were used.

The technical state of each set $\mathrm{O}=\left\{\mathrm{O}_{\mathrm{i}}: \mathrm{i}=1,2,3, \ldots, \mathrm{M}\right\}$ entering the repair fund of car vehicle component parts and special oil and gas field equipment is characterized by a set of structural, dimensional and diagnosis parameters. Their values are determined at the stage of pre-repair diagnosis with centralized repair according to their technical state. Определенные сочетания данных параметров и их значений означают наличие или отсутствие дефектов агрегатов Certain combinations of these parameters and their values mean the presence or absence of defects in vehicle component parts [8,9].

We introduce the concept of "Generalization ability". It is the property of a neural network acquired in the process of training to produce correct results for any new input combinations which did not participate in the learning process.

If artificial neural networks show a high percentage of correct results not only for the training sample, but also for new, previously unknown examples, it is believed that it has acquired the generalization ability.

In the case where a high percentage of correct results is provided only for the training sample, and is often mistaken on test cases, then we can conclude that the neural network did not have the generalization ability.

Let us assume that the amount of repair work variety at the centralized repair according to the technical condition is a previously known value of $\mathrm{Z}$, and $\mathrm{X}$ is the combination of the structural, dimensional and diagnostic parameters of the units coming in for repair. Deviations in the parameter values from the nominal testify to the presence of defects in the vehicle component parts $\mathrm{Y}$. In this case, the neural network classification problem is reduced to the construction of an algorithm $\Theta$, where at the initial stage there is a classification $\Theta: X \rightarrow Y$, there is a distribution of the vehicle component parts for the repair work variety, depending on the revealed combinations of defects: $\Theta: Y \rightarrow Z$, where $y \in Y$ and $z \in Z$, based on the neural network generalization ability, acquired in the learning process

When building a neural network classifier, first of all, it is necessary to determine the complexity of the separation of objects into classes. To simplify the classification problem, it is necessary to achieve a linear separation of research objects.

Since the problem posed implies more than two classes for the distribution of vehicle component parts between them, the most rational way of generating the output signals will be the set of the vector components. In other words, each possible defect will have its own output signal, and the presence of a defect or its absence will say 0 or 1 at the corresponding output. It is very important to achieve as close to 0 or 1 values. It is necessary to pre-process the input data.

\subsection{The technique of preliminary processing of artificial neural networks input data}

To increase the efficiency and reduce the training time of a neural network, the data arriving to the input layer of the network must be typed and lie in a certain range, so the training data set need to be scaled.

Typically, the training data set is fed to the input layer in the form of binary values, integers, floating-point numbers, etc. Their spread should be in a certain range, which depends on the type of the used activation function. However, most often, incoming signals 
have a wide spread of values on each neuron of the input layer, which significantly reduces the cognitive abilities of artificial neural networks.

The values range of the sigmoid function is in the range $[0 ; 1]$, therefore it is compressive one (Figure 5).

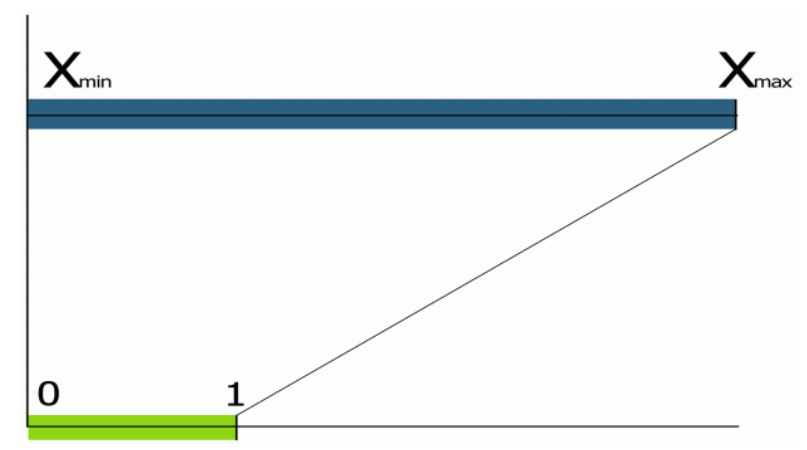

Fig. 5. Scaling of initial data in the values range of the sigmoidal activation function

Considering the mentioned above, an algorithm for training a neural network with a sigmoid as an activation function was developed.

During the operation of the algorithm for scaling the original data, the transfer to the activation function is carried out in the optimal form. This algorithm is universal, so when it is used for an activation function different from a sigmoidal one, the scaling interval and, possibly, the type of rationing changed in step 3.

Linear normalization is used for dense filling of the interval of variable $\mathrm{x}_{\mathrm{i}}$ with values, because it is oriented to boundary values $\left(\mathrm{x}_{\min }, \mathrm{x}_{\max }\right)$.

\subsection{The technique of neural network classification of vehicle component parts taking into account their actual technical condition}

The methodology of neural net classification of vehicle component parts under the centralized repair according to the technical condition is divided into two stages.

- At the first stage, defects of vehicle component parts are detected depending on combinations of structural, dimensional, and other parameters whose deviations from the allowed values indicate the presence of a defect.

- At the second stage, the vehicle component parts, according to the results of the revealed combinations of defects, are distributed among the repair work complexes, depending on the developed distribution criterion.

The mathematical model does not have a clearly expressed form, therefore the methodology for distributing the vehicle component parts according to the repair work variety depending on the detected defects in the centralized repair according to the technical condition is shown in block schematic diagrams (Figures 9 and 10).

\section{Results}

\subsection{Algorithm for constructing an artificial neural network for identifying combinations of defects in the repair fund units.}


To identify combinations of defects in the repair fund units using an artificial neural network at the centralized repair according to the technical condition, an algorithm was formed.

- Step 1. Database (DB) of combinations of structural, dimensional and other parameters (DB1) is compiled on the basis of the experimental data. In this case, the database should be divided into two parts, based on the selected percentage: the training sample (based on it, the network is trained, so this set has the largest number of examples from the database) and the test sample (on which basis the quality of defects in the vehicle component parts is assessed)

- Step 2. The normalizing takes place according to the graph in Fig.

- Step 3. Initially, the number of neurons in the hidden layer $=2$. To determine the optimal number, we will use the method of incrementing their number until an optimal value is reached at which the test data set will be successfully recognized. To determine the upper limiting value of the number of neurons in the hidden layer, we use the formula (6).

- Step 4. If the number of neurons in the hidden layer has not reached its set limit value $R$, then the next step is taken. Otherwise, it is unlikely that a further increase in the number of neurons will improve the quality of artificial neural networks training, so increasing the number of neurons will improve the quality of artificial neural networks training, therefore it's necessary to go back to step 1 and reformat the initial database of parameter combinations (DB1).

- The artificial neural networks training takes place by the method of back propagation of the error. The number of neurons of the input layer is taken equal to the number of parameters indicating the presence of defects in certain assembly nodes. The number of neurons in the output layer is equal to the number of possible defects of the subassembly in question.

All output signals of the last layer of the network make sense to transfer into a logical data type, which takes the values: 0 - no defect, 1 - defect. The combination of output signals $\mathrm{Y}_{1} \ldots \mathrm{Y}_{\mathrm{L}}$ will be the basis for claiming the presence of a defect.

- Step 6. It is estimated that the neural network's property, acquired in the process of training, reveals previously unknown defects (from the test sample that did not participate in the learning process), formed as a result of various combinations of parameters according to the structural-effect model of the interconnection of the aggregate parameters.

- Step 7. If the test sample is fully recognized, it can be concluded that the artificial neural networks has acquired the generalization ability. In this case, a transition to step 9 takes place. Otherwise, the artificial neural networks either did not acquire the generalization ability, or the error value is still high. In this case a transition to step 8 takes place.

- Step 8. There is an increase in the number of neurons $\mathrm{L}=\mathrm{L}+1$ and go to the beginning of step 4 .

- Step 9. Upon completion of the algorithm at the output, we get test combinations of defects in vehicle component parts. A certain complex of repair works is assigned to each such combination. The results are written to a separate database (DB2). 


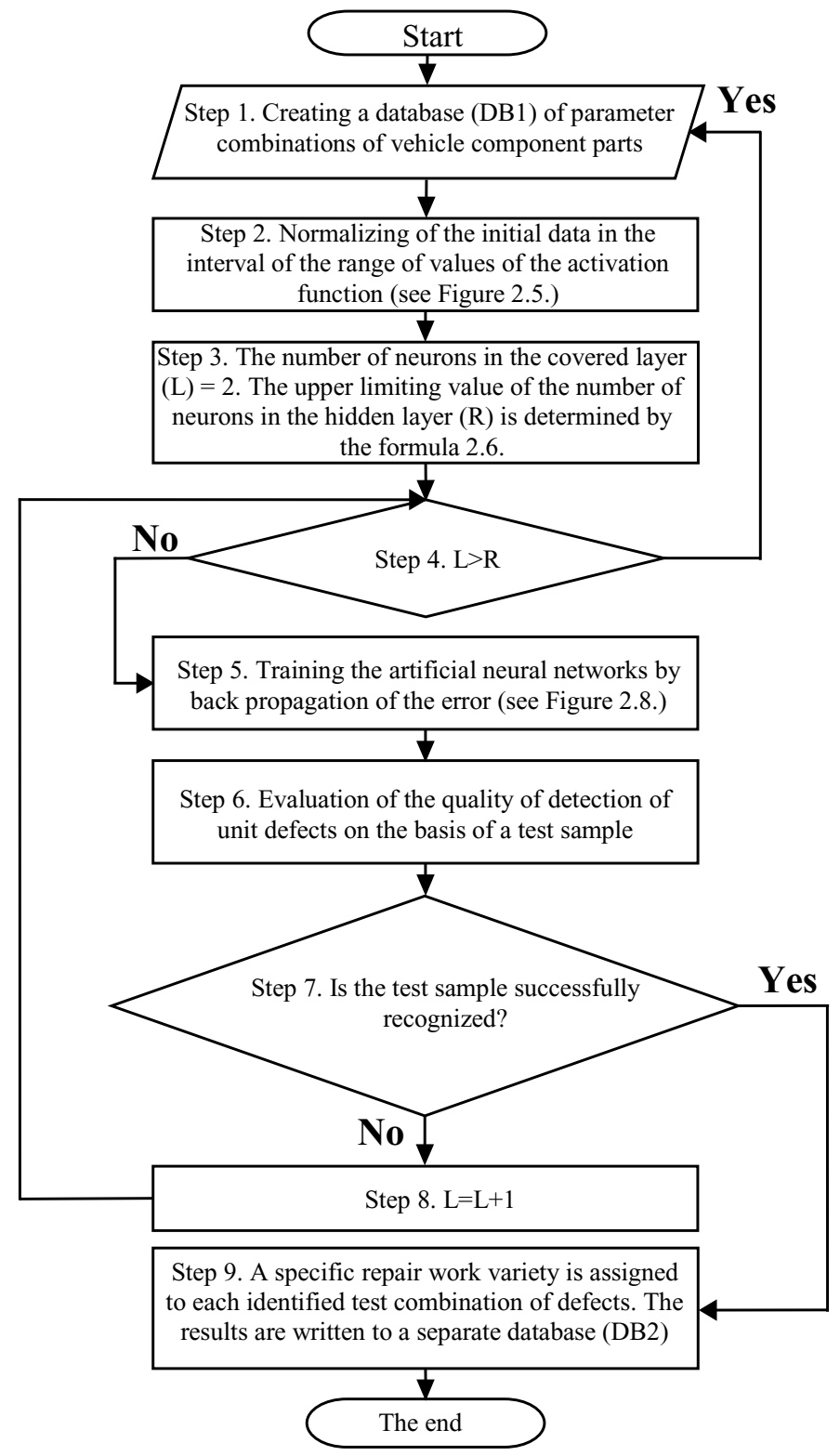

Fig. 6. A block schematic diagram of an artificial neural networks construction algorithm for solving the problem of detecting of vehicle component parts defects

\subsection{Algorithm of neural network classification of vehicle component parts depending on the revealed combinations of defects}

To distribute the repair fund assemblies to the repair works at the centralized repair according to the technical condition, the database (DB2), formed as a result of the work of the previous algorithm, is used, which lists all the revealed combinations of defects in vehicle component parts.

In Fig. 7 in the form of a block schematic diagram, an algorithm for constructing a neural network classifier is presented for solving the problem of optimal distribution of car 
vehicle component parts and special oil and gas equipment for repair work variety. It should be noted that the steps where the input data are normalized, the determination of the optimal number of neurons in the hidden layer, and the procedure for learning by the method of back propagation of the error are analogous to the corresponding steps of the algorithm in Fig. 6.

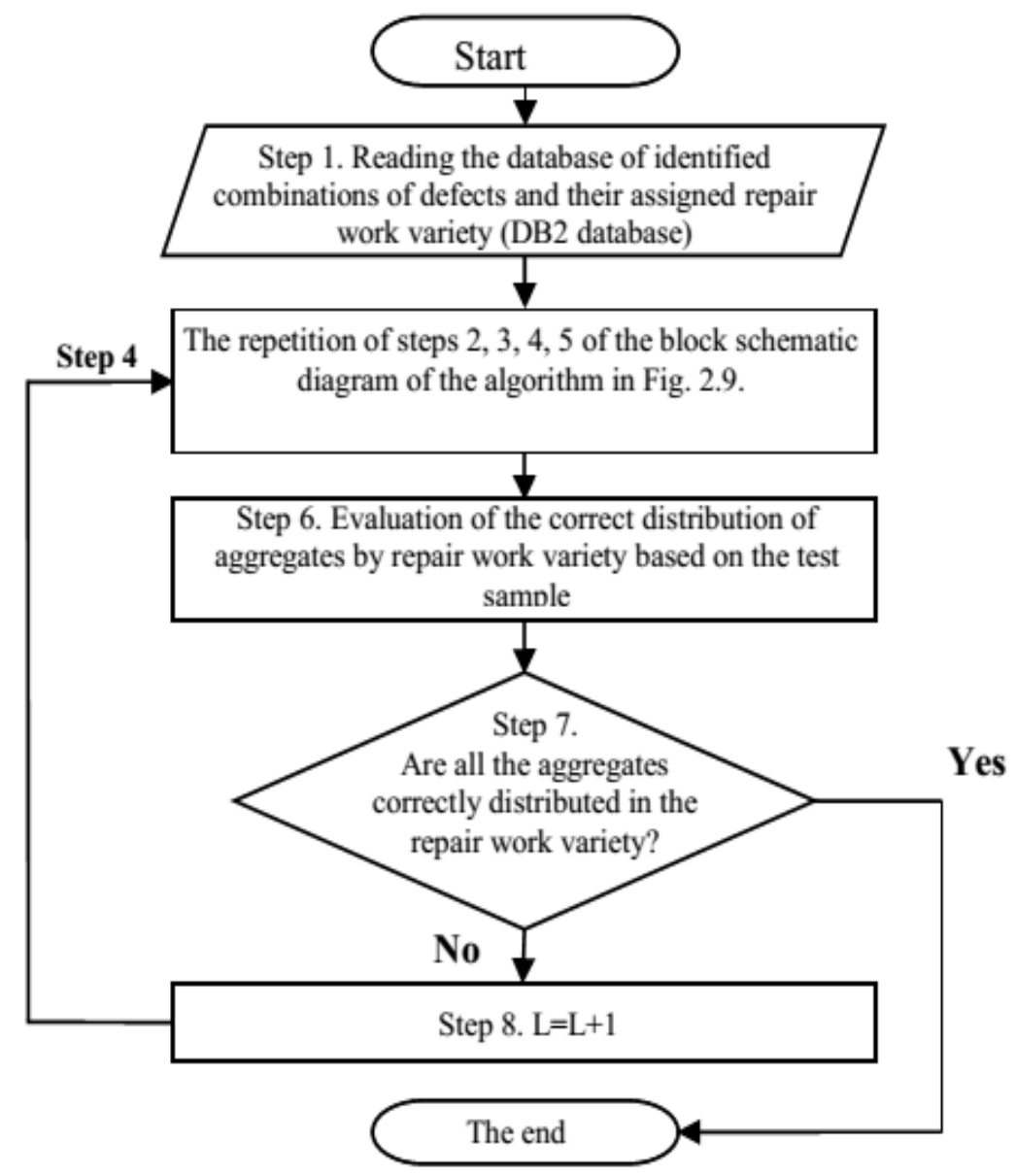

Fig. 7. Block schematic diagram of the algorithm for constructing artificial neural networks for the solution of the problem of neuro net classification of vehicle component parts by repair work variety

\section{Conclusion}

1.The use of artificial neural networks for detection of defects in aggregates and their subsequent distribution by repair work variety, allows to avoid mistakes of the 1 st (false defect) and the second type (defect passing), because when the neural network approach, the error function depends on the correctly constructed artificial neural networks architecture for the task as well as on the quality and quantity of data in the training sample.

2. Introduction of an additional moment $\boldsymbol{\mu}$ in the backward propagation algorithm of the error makes it possible to significantly improve the learning efficiency. 
3. The presented methodology using artificial neural network models is quite flexible, so it can be easily adapted in addition to identifying defects in car vehicle parts and special oil and gas equipment, for aviation, maritime and other transport, where repairs are carried out according to the actual technical condition.

\section{References}

1. V.I. Karagodin, Modeling of technological processes and car repair systems: collection of scientific papers. Moscow: MADI, 14-23 (1987)

2. D. Rutkovskaya, Neural networks, genetic algorithms and fuzzy systems. Moscow: Hot line - Telecom, 452 p., (2006).

3. V.A. Korchagin, V.N. Krasovskiy, New technologies for the oil and gas region: proc. of the All-Russian scientific and practical conference. 1; Tyumen: TyumGNGU, 34-37, (2012)

4. L.N. Yasnitsky Introduction to Artificial Intelligence. Moscow: Publishing Center "Academy", 176 p., (2008)

5. A.A., Uskov, A.V. Kuzmin, Intelligent control technologies. Artificial neural networks and fuzzy logic. Moscow: Hot line - Telecom, 144 p., (2004)

6. U.S. McCulloch, V. Pitts,Logical Calculus of Ideas Relating to Nervous Activity. In coll.: "Avtomaty". Moscow: Izd-vo inostr. lit., (1956).

7. D. Rutkovskaya, op. cit.

8. V.N. Krasovsky, Collected scientific works SWorld. "Modern Directions of Theoretical and Applied Research 2012". Issue 1. 1. Odessa: KUPRIENKO, 77-82, (2012)

9. V.V. Poptsov, V.N. Krasovsky, V.A. Korchagin, V-Biosciences biotechnology research, Asia, 12(2), 1857-1866, (2015) 